\title{
Pyrano- und Naphthopyrano[3,2-b]indol-Derivate ${ }^{1)}$
}

\author{
Fritz Eiden * und Klaus Th. Wanner \\ Institut für Pharmazie und Lebensmittelchemie der Universităt München, Sophienstraße 10, \\ 8000 München 2 \\ Eingegangen am 13. März 1984
}

Die Pyrano[3,2-b]indole 4a-4c lassen sich durch regioselektive Reaktion des Tetrahydro-3-pyranons (3) mit den Phenylhydrazinen 6-6e gewinnen, das Naphthopyrano[3,2-b]indol 24 entsteht durch Umsetzung des Naphthopyranons 23 mit 6a. 23 labt sich aus dem Dihydropyranon 16 und dem Isobenzofuran 21 durch Cycloaddition und Dehydratisierung der so gewonnenen endolexoEpoxynaphthopyranone 22a/22ab herstellen.

\section{Pyrano- and Naphthopyrano[3,2-b]indole Derivatives}

The pyrano [3,2-b]indoles $40-4 c$ can be obtained by regioselective reactions of tetrahydro-3-pyranone (3) with the phenylhydrazines 6a-6e. Reaction of naphthopyranone 23 with 6 yields the naphthopyrano[2,3-b]indole 24. Compound 23 can be obtained from dihydropyranone 16 and isobenzofurane $\mathbf{2 1}$ by cycloaddition and dehydration of the resulting endolexo-epoxynaphthopyranones $22 \mathrm{a} / 22 \mathrm{~b}$.

Es gibt Pyrano[3,4-b]indole mit bemerkenswerten biologischen Eigenschaften. Dabei sind vor allem die Substituenten in 1-Stellung von Bedeutung: Essigsäure-Derivate, z.B. Prodolsäure (1a) und Etodolsăure (1b), kơnnen entzündungshemmende Wirkungen zeigen ${ }^{2)}$ und bei Dialkylaminopropylabkömmlingen, 2.B. 1c, hat man antidepressive Eigenschaften gefunden ${ }^{3}$. Auch Pyrano[3,2-b]indole sind in diesem Zusammenhang interessant; die Pyranoindol-carbonsäure 2 2.B. soll antiallergische Wirkungen besitzen").

Wir haben die Eignung von Tetrahydro-3-pyranon 3 zur Darstellung pyrananellierter Heterocyclen untersucht ${ }^{3}$ ) und dabei geprüt, ob 3 sich auch zur Synthese von Pyrano-indolen verwenden läBt. Durch Umsetzungen von 3 mit 6a oder $6 b$ in Essigsäure bei Raumtemperatur oder mäBigem Erwärmen entstanden - Analysen und Spektren

0365-6233/65/0606-0548 5 02.500

- VCH Verlagreseclbchaf mbH, D-6900 Weinbeim. 1985 
<smiles>[Z12]c1cccc2c3c([nH]c12)C([R1])([R1])COC3</smiles>

\begin{tabular}{l|lll}
1 & $\mathrm{R}^{2}$ & $\mathrm{R}^{2}$ & $\mathrm{R}^{3}$ \\
\hline $\mathbf{2}$ & $\mathrm{CH}_{2} \mathrm{COOH}$ & $\mathrm{C}_{3} \mathrm{H}_{7}$ & $\mathrm{H}$ \\
b & $\mathrm{CH}_{2} \mathrm{COOH}$ & $\mathrm{C}_{2} \mathrm{H}_{5}$ & $\mathrm{C}_{2} \mathrm{H}_{5}$ \\
C & $\left(\mathrm{CH}_{2}\right)_{3} \mathrm{~N}\left(\mathrm{CH}_{3}\right)_{2}$ & $\mathrm{CH}_{3}$ & $\mathrm{H}$
\end{tabular}<smiles>Cn1c2ccccc2c2oc(C(=O)O)cc(=O)c21</smiles>

2

zufolge - Pyranoindol-Derivate. Unter gleichen Bedingungen reagierte 3 mit 60 zum Phenylhydrazon 7; zur Synthese des entsprechenden Indols mußte die Reaktionszeit verlängert und die Temperatur erhöht werden.

Mit Hilfe der ' $\mathrm{H}$-NMR-Spektren ließ sich zeigen, daß bei diesen Umsetzungen regioselektiv die [3,2-b]-Isomere $4 a, 4 b$ und $4 c$ entstanden sind (s. Tab. 1); dabei sind insbesondere die $\mathrm{O}-\left(\mathrm{CH}_{2}\right)_{3}$-Signale charakteristisch, die eine $[3,4-b]$-Anordnung (5) ausschließen.

Tab.1: 'H-NMR-Daten von 4a, $4 \mathrm{~b}$ und $4 \mathrm{c}, 8$-Werte $(\mathrm{J}=\mathrm{Hz})$, in $\mathrm{CDCl}_{3}$

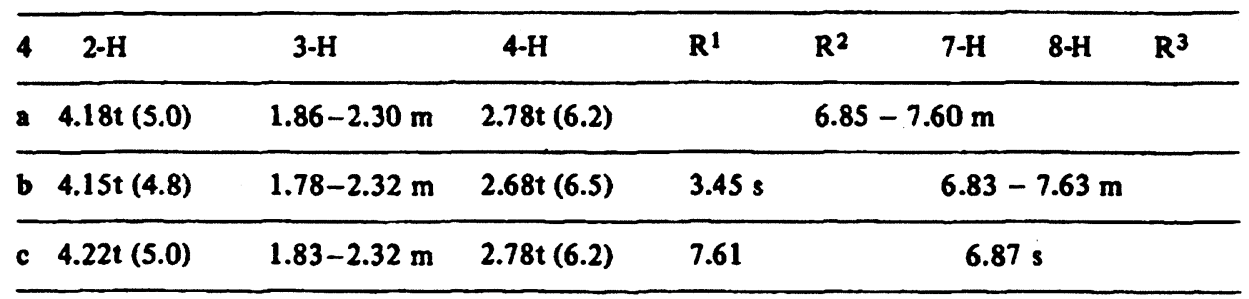<smiles>[R]c1ccc([R2])c(N([R1])N)c1</smiles><smiles></smiles>

\begin{tabular}{l|lll}
$4 / 6$ & $\mathrm{R}^{1}$ & $\mathrm{R}^{2}$ & $\mathrm{R}^{3}$ \\
\hline $\mathbf{2}$ & $\mathrm{H}$ & $\mathrm{H}$ & $\mathrm{H}$ \\
$\mathrm{b}$ & $\mathrm{CH}_{3}$ & $\mathrm{H}$ & $\mathrm{H}$ \\
$\mathrm{C}$ & $\mathrm{H}$ & $\mathrm{Cl}$ & $\mathrm{Cl}$
\end{tabular}


Mit $8 \mathrm{and}$ ub sind entsprechende Indolsynthesen bereits durchgeführt worden: Während die Umsetzungen des Tetrahydro-thiopyranons 8a mit zum Thiopyrano[3,2-b]indol 9 führte $e^{6)}$, entstand bei der Reaktion des 3-Piperidons 86 mit 4-Methylhydrazin (6d) das Tetrahydropyridino[3,4-b]indol $10^{n}$.

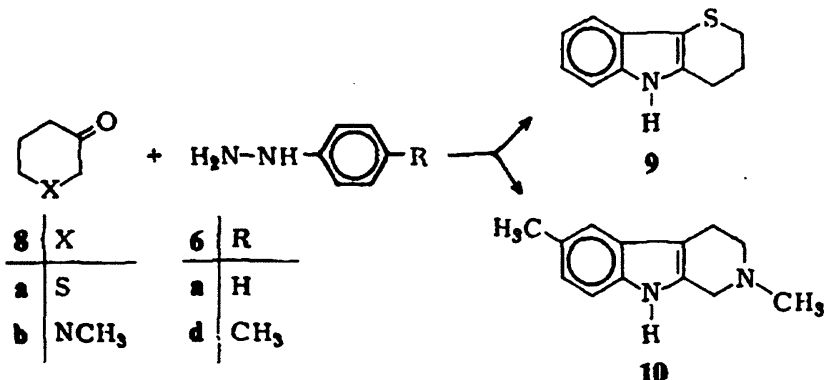

Wir haben weiterhin versucht, $[3,4]$-naphthoanellierte Pyrano[3,2-b]indole herzustellen, also Derivate von 11; dabei hat uns die Yohimban- bzw. Oxayohimbanstruktur 12a bzw. 12b, die in wirksamen Indolalkaloiden steckt ${ }^{8}$, als Modell gedient.<smiles>c1ccc2cc3c(cc2c1)COc1c-3[nH]c2ccccc12</smiles><smiles>[X]C1CCC2CN3CCc4c([nH]c5ccccc45)C3CC2C1</smiles>

Ausgangssubstanz hierzu war das Dihydro-3-pyranon 16, das wir in Analogie zu einem von Skinnemoen und Undheim angegebenen Verfahren zur Herstellung 5-substituierter Dihydro-3-pyranone" synthetisierten:

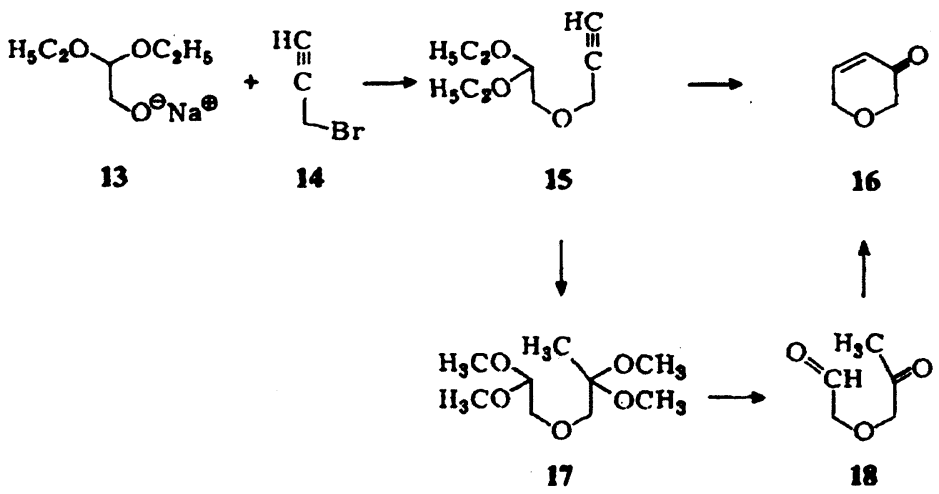


Das Natriumsalz des Hydroxyacetaldehydacetals 13 wurde mit Propargylbromid (14) zum Ether 15 umgesetzt, der sich in Methanol durch Zugabe eines $\mathbf{H g}$ (II)-Katalysators und von Ameisensäure in einem Eintopfverfahren zum Pyranon 16 umsetzen ließ. Zwischenprodukte wie das Tetramethoxy-Derivat 17 und der Oxopropoxyacetaldehyd 18 konnten isoliert werden.

Das 'H-NMR-Spektrum des Reaktionsproduktes stimmt mit der Struktur 16 gut überein (Olefinsignale bei $\delta=6.10$ und 7.12 ppm und $\mathrm{CH}_{2}$-Signale bei 4.35 und $4.13 \mathrm{ppm}$ ). Außerdem ließ sich 16 durch katalytische Hydrierung in das Pyranon 3 überführen und mit 4-Nitrophenylhydrazin (19a) sowie Semicarbazid (19b) unter Bildung der Azomethine 20a und $20 \mathrm{~b}$ kondensieren.

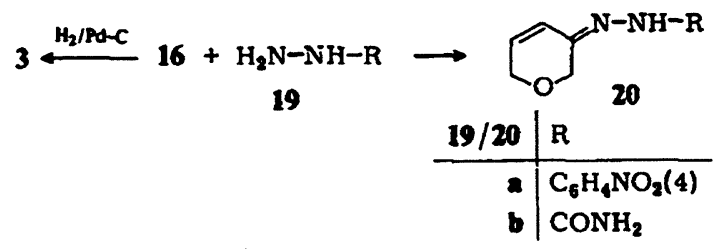

Das Enon 16 sollte nun über eine [4+2]-Cycloaddition in ein Naphtho-Derivat übergeführt werden und das gelang auch mit dem Diphenyl-benzoisofuran 21. Wir erhielten ein - durch fraktionierte Kristallisation auftrennbares - Gemisch der endo/ exo-Isomere 22a und 22b (Racemate). Durch Erwärmen mit Phosphorsäure entstand das Naphthopyranon 23 in 83proz. Ausbeute.

$16+$

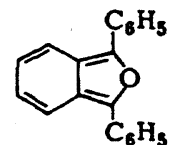<smiles>CC(C)C</smiles>

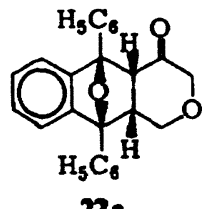

22.

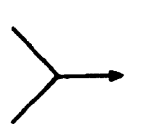

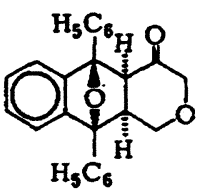

$22 b$<smiles>CCc1c2c(c([14CH3])c3ccccc13)C(=O)COC2</smiles>

23

23 schließlich reagierte mit Phenylhydrazin (6a) - wiederum in sehr guter Ausbeute zum Naphthopyranoindol 24. 
Arch. Pharm.

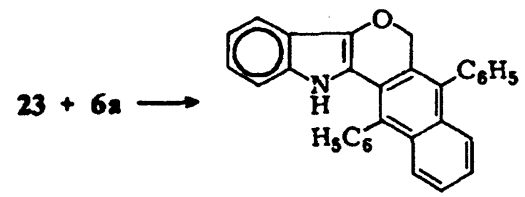

24

Dem Fonds der Chemischen Industrie danken wir für die finanzielle Unterstützung unserer Untersuchungen, Frau $\boldsymbol{A}$. Kärtner für die engagierte Mitarbeit beim Experimentieren.

\section{Experimenteller Teil}

Schmp. (nicht korr.): Schmelzpunktsbestimmungsapparat nach Dr. Tottoli und Opfer-SchaumGerăt. 'H-NMR-Spektren: T-60 und A-60 (Varian); 8 -Skala (ppm), TMS int. Stand. ${ }^{13} \mathrm{C}$. NMR-Spektren: WP 80 (Bruker), 8-Skala (ppm), TMS int. Stand. MS: CH 7 (Varian). IR-Spektren: Acculab 6 (Beckman). Flüssigkeiten als Film, Feststoffe als KBr-PreBling. CHN-Analysen: CHN-Analyzer Modell 185 (Hewlett-Packard); CHN-Rapid (Heraeus). SC: Kieselgel 60, KorngröBe 0.063-0.200 mm (Fa. Merck, Nr. 7734). Schutzgas: $\mathrm{N}_{2}$.

\section{5,6-Dihydro-2H-pyran-3(4H)-on (3)}

$0.98 \mathrm{~g}(10 \mathrm{mmol}) 16$ wurden in $10 \mathrm{ml}$ absol. Ethanol gelost und mit $0.03 \mathrm{~g} 10$ proz. Pd/C versetzt. Es wurde unter Normaldruck bei Raumtemp. hydriert, dann filtriert und $2 \mathrm{ml}$ Ameisensāure zugesetzt, um gebildetes Acetal zu spalten. Nach 30 min wurde i. Vak. abgedampft und der flüssige Rúckstand mit $15 \mathrm{ml}$ Methylenchlorid und $3 \mathrm{~g}$ basischem Aluminiumoxid (Korngröße 0.063-0.200 mm, Aktivitätsstufe I, Merck Nr. 1076) versetzt. Dann wurde filtriert und i. Vak. destilliert. Farblose Flüssigkeit, Sdp. ${ }_{11} 51-54^{\circ}\left(\right.$ Lit. $^{10)}$ : Sdp. $\left.{ }_{15} 61^{\circ}\right)$, Ausb. $0.3 \mathrm{~g}(30 \%)$.

\section{2,3,4,5-Tetrahydro-pyrano[3,2-b]indol (4a)}

Eine Lösung von $1.08 \mathrm{~g}(10 \mathrm{mmol}) 6 \mathrm{a}$ und $1.0 \mathrm{~g}(10 \mathrm{mmol}) 3 \mathrm{in} 6 \mathrm{ml}$ Eisessig wurde kurz erwärmt und $36 \mathrm{~h}$ bei Raumtemp. gerührt. Nach Zusatz von Diethylether wurde mit Wasser gewaschen, getrocknet (Natriumsulfat) und i. Vak. eingeengt. Aus Essigester gelbe, an der Luft zersetzliche Kristalle,

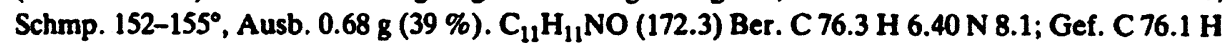
6.41 N 8.1 Mol.-Masse 173 (ms). - IR: 3380, 1615, 1330, $755 \mathrm{~cm}^{-1}$.

\section{2,3,4,5-Tetrahydro-5-meti:yl-pyrano[3,2-b]indol (4b)}

$\mathrm{Zu} 1.22 \mathrm{~g}(10 \mathrm{mmol}) 6 \mathrm{~b}$ und $6 \mathrm{ml}$ Eisessig wurde unter Kühlung (Wasserbad) $1.0 \mathrm{~g}$ (10 mmol) 3 gegeben, dann $18 \mathrm{~h}$ bei Raumtemp. gerührt, mit Diethylether verdünnt, mit Wasser ausgeschüttelt, getrocknet (Natriumsulfat) und i. Vak. eingeengt. Nach Destillation bei $10^{-4}$ Torr $\left(100-120^{\circ}\right.$ Badtemp.) und Kristallisation aus Methanol gelbe, an der Luft zersetzliche Kristalle, Schmp. 49-50, Ausb. $1.1 \mathrm{~g}(59 \%) . \mathrm{C}_{12} \mathrm{H}_{13} \mathrm{NO}$ (187.2) Ber. C77.0H 7.00 N 7.5; Gef. C76.9 H 6.98 N 7.6 Mol.-Masse 187 (ms). - IR: 2965, 1590, $1380,730 \mathrm{~cm}^{-1}$.

\section{6,9-Dichlor-2,3,4,5-tetrahydro-pyrano[3,2-b]indol (4c)}

Eine Mischung aus $1.77 \mathrm{~g}(10 \mathrm{mmol}) 6 \mathrm{ce}, 1.1 \mathrm{~g}(11 \mathrm{mmol}) 3$ und $6 \mathrm{ml}$ Eisessig wurde $12 \mathrm{~h}$ bei $60-70^{\circ}$ Badtemp. gerührt. Nach dem Abkühlen wurde mit Diethylether verdünnt, mit Wasser gewaschen, getrocknet (Natriumsulfat), i. Vak. eingeengt und der Rückstand aus Methanol umkristallisiert. 
Dann wurde sc gereinigt (Füllhöhe $=15 \mathrm{~cm}, \boldsymbol{O}=28 \mathrm{~cm}$, Laufmittel $=$ Methylenchlorid, 15 ml-Fraktionen). Die Fraktionen 4-7 wurden i. Vak. eingedampft und der Rückstand aus Cyclohexan umkristallisiert. Hellgelbe Nadeln, Schmp. 135-137.5 , Ausb. 0.18 g (17 \%). $\mathrm{C}_{11} \mathrm{H}_{9} \mathrm{Cl}_{2} \mathrm{NO}$ (242.1) Ber. C 54.6 H 3.75 N 5.8 Cl 29.3; Gef. C 54.5 H 3.77 N 5.7 Cl 29.2 Mol.-Masse 242 (ms). - IR: 3320, $1625,1600,1085 \mathrm{~cm}^{-1}$.

\section{5,6-Dihydro-2H-3(4H)pyranon-2', $5^{\prime}$-dichlorphenylhydrazon (7)}

$1.77 \mathrm{~g}(10 \mathrm{mmol}) 6 \mathrm{c}$ wurden zusammen mit $1.0 \mathrm{~g}$ (10 mmol) 3 in $10 \mathrm{ml}$ Eisessig gelöst. Dann wurde 0.5 h bei $70^{\circ}$ und $12 \mathrm{~h}$ bei Raumtemp. gerührt, der Niederschlag abgetrennt, das Filtrat mit Diethylether verdünnt, mit Wasser gewaschen, getrocknet (Natriumsulfat) und i. Vak. eingeengt. Der Rückstand wurde zusammen mit dem 1. Niederschlag aus Methanol umkristallisiert. Hellgelbe Kristalle, Schmp.

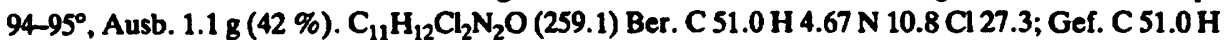
4.62 N 10.7 Cl 27.4 Mol.-Masse 259 (ms). - IR: $3480,1660,1100 \mathrm{~cm}^{-1}$. - ${ }^{1} \mathrm{H}-\mathrm{NMR}\left(\mathrm{CDCl}_{3}\right): 1.70-2.18$ $(\mathrm{m}, 2 \mathrm{H}), 2.57(\mathrm{t}, \mathrm{J}=6.5 \mathrm{~Hz}, 2 \mathrm{H}), 4.26(\mathrm{~s})$ und 4.37 (s) (Relation $1.5: 0.3$, zusammen $2 \mathrm{H}$, Signale der $E_{-,} Z$-Isomere), 6.73 (dd, J $\left.=9 / 2.5 \mathrm{~Hz}, 1 \mathrm{H}\right), 7.19$ (d, J $\left.=9 \mathrm{~Hz}, 1 \mathrm{H}\right), 7.50(\mathrm{~d}, \mathrm{~J}=2.5 \mathrm{~Hz}, 1 \mathrm{H}$ ), 7.42-7.69 (1H, $\mathrm{D}_{2} \mathrm{O}$-Austausch).

\section{2-(3-Propinyloxy)-acetaldehyddiethylacetal (15)}

Eine Suspension von $14.4 \mathrm{~g}(0.60 \mathrm{~mol})$ Natriumhydrid (50proz. Dispersion in $\mathrm{Ol})$ in $200 \mathrm{ml}$ absol. THF wurde bei Raumtemp. unter Rühren tropfenweise mit einer Lösung von $80.4 \mathrm{~g}$ (0.60 mol) Glykolaldehyddiethylacetal in $400 \mathrm{ml}$ absol. THF versetzt. Die Mischung wurde $2 \mathrm{~h}$ bei Raumtemp. gerührt. Dann wurden $95.4 \mathrm{~g}(0.8 \mathrm{~mol}) 14$ (80proz. Lösung in Toluol) so zugegeben, daß der Ansatz schwach siedete. AnschlieBend wurde 12 h rückfließend erhitzt. Unverbrauchtes Natriumhydrid wurde durch Zugabe von Ethanol zersetzt, das gebildete Natriumbromid abgesaugt und das Filtrat $i$. Vak. destilliert. Farblose Flüssigkeit, Sdp.10 82-87 , Ausb. 86.1 g (83 \%). $\mathrm{C}_{9} \mathrm{H}_{16} \mathrm{O}_{3}$ (172.2) Ber. C 62.8 H 9.36; Gef. C 62.8 H 9.36 Mol.-Masse 172 (ms). - IR: 2975, $1105 \mathrm{~cm}^{-1}$. - ${ }^{1} \mathrm{H}-\mathrm{NMR}\left(\mathrm{CDCl}_{3}\right)$ : $1.22(\mathrm{~s}, 6 \mathrm{H}), 2.45(\mathrm{t}, \mathrm{J}=2.3 \mathrm{~Hz}, \mathrm{HH}), 3.33-3.87(\mathrm{~m}, 6 \mathrm{H}), 4.17(\mathrm{~d}, \mathrm{~J}=2,3 \mathrm{~Hz}, 2 \mathrm{H}), 4.62(\mathrm{t}, \mathrm{J}=5 \mathrm{~Hz}$, 1H).

\section{H-3(6H)-pyranon (16) und (2-Oxopropoxy)-acetaldehyd (18)}

Zur Darstellung des Katalysators wurde eine Mischung aus $2.40 \mathrm{~g}$ rotem Quecksilber(II)-oxid, $0.24 \mathrm{~g}$ Trichloressigsäure, $4.0 \mathrm{ml}$ Bortrifluorid-Etherat und $5.0 \mathrm{ml}$ Methanol bis zum Verschwinden der roten Farbe erhitzt. Der Katalysator wurde in $60 \mathrm{ml}$ absol. Methanol suspendiert und mit $60.3 \mathrm{~g}(0.35$ mol) 15 (in $40 \mathrm{ml}$ absol. Methanol) so versetzt, daB die Innentemp. zwischen 40 und $50^{\circ}$ betrug. Es wurde noch 2 h bei Raumtemp. gerührt, Quecksilber durch Zusatz von Natriumborhydrid abgeschieden und dann mit $200 \mathrm{ml}$ Pentan verdünnt, filtriert und das Filtrat i. Vak. vom Lösungsmittel befreit. Der lüssige Rückstand wurde mit 21 Ameisensäure versetzt. Nach 3 min wurden 21 Chloroform zugefügt, und man lieB die Mischung 88 h rïckflieBend sieden. Dann wurde i. Vak. weitgehend abgedampft, nach Zusatz von $200 \mathrm{ml}$ Diethylether, $50 \mathrm{ml}$ Pentan und $30 \mathrm{ml}$ Methanol Unlösliches abgetrennt, die Mischung über Natriumsulfat und basischem Aluminiumoxid (KorngröBe 0.063-0.200 mm, Aktivitătsstufe I, Merck Art. Nr. 1076) filtriert und das Filtrat i. Vak. vom Lösungsmittel befreit. Ein Teil des flüssigen Rückstandes lieB sich bei 0.05 Torr und $40^{\circ}$ Badtemp. abdampfen und in einer Kühlfalle (Methanol/Trockeneis) auffangen. Aus dem Kondensat wurde durch Destillation 16 gewonnen. Farblose Flussigkeit; Sdp. 34 51-53', Ausb. 8.75 \& (26 \%). $\mathrm{C}_{5} \mathrm{H}_{6} \mathrm{O}_{2}$ (98.1) Ber. C 61.2 H 6.16; Gef. C 61.0 H 6.38 Mol.-Masse 98 (ms). - IR: 1690, $1675 \mathrm{~cm}^{-1}$. ${ }^{13}$ C-NMR (d $\left.{ }_{6}-D M S O\right): 64.4$ (t), 71.7 (t), 126.0 (d), 150.3 (d), 194.5 (s). 
Aus dem beim Übertreiben verbleibenden Rückstand (s. O.) ließ sich 18 bei $10^{-3,5}$ Torr und $91^{\circ}$ Badtemp. abdestillieren. Farblose, sich rasch gelbfärbende Flüssigkeit, Ausb. $1.2 \mathrm{~g}(3 \%) . \mathrm{C}_{9} \mathrm{H}_{8} \mathrm{O}_{3}$ (116.1) Ber. C 51.7 H 6.94; Gef. C 51.6 H 6.95 Mol.-Masse 116 (ms).

\section{(2-Oxopropoxy)-acetaldehyd (18)}

$25.0 \mathrm{~g}(120 \mathrm{mmol}) 17$ und $500 \mathrm{ml}$ Ameisensăure wurden $2 \mathrm{~h}$ bei Raumtemp. gerührt. Es wurde i. Vak. abgedampft und der Rückstand destilliert. Farblose Flüssigkeit, Sdp.0.4 57-59 , Ausb. 9.1 g (65 \%). $\mathrm{C}_{5} \mathrm{H}_{8} \mathrm{O}_{3}$ (116.1) Ber. C 51.7 H 6.94; Gef. 51.5 H 7.35 Mol.-Masse 116 (ms). - IR: $1715 \mathrm{~cm}^{-1}$. ${ }^{1} \mathrm{H}-\mathrm{NMR}\left(\mathrm{CDCl}_{3}\right): 2.19(\mathrm{~s}, 3 \mathrm{H}), 4.28(\mathrm{~s}, 4 \mathrm{H}), 9.85(\mathrm{~s}, 1 \mathrm{H})$.

\section{1,1,5,5-Tetramethoxy-3-oxahexan (17)}

Aus $4.0 \mathrm{~g}$ rotem Quecksilber(II)-oxid wurde, wie bei 16 beschrieben, der Katalysator hergestellt und in $300 \mathrm{ml}$ absol. Methanol suspendiert. $25.8 \mathrm{~g}$ (150 mmol) 15 in $40 \mathrm{ml}$ absol. Methanol ließ man so zutropfen, daB die Temp. 40 bis $45^{\circ}$ betrug. Nach $9 \mathrm{~h}$ wurden $25 \mathrm{~g}$ Natriumboranat so zugegeben, daB die Temp. $40^{\circ}$ nicht überstieg. Es wurde filtriert, i. Vak. eingeengt und mit Diethylether und Wasser versetzt. Nach Abtrennen der organischen Phase wurde mit Ether extrahiert. Die vereinigten organischen Extrakte wurden gewaschen, getrocknet und i. Vak. eingeengt. Nach Destillation farblose Flüssigkeit, Sdp. ${ }_{10}$ 103-106 $^{\circ}$, Ausb. $24.07 \mathrm{~g}$ (77\%). $\mathrm{C}_{9} \mathrm{H}_{20} \mathrm{O}_{5}$ (208.2) Ber. C51.9 H 9.68; Gef. C 51.8 H 9.61 Mol.-Masse 208 (ms). - IR: 1460, $1375 \mathrm{~cm}^{-1}$. - ${ }^{1} \mathrm{H}-\mathrm{NMR}\left(\mathrm{CDCl}_{3}\right): 1.36$ (s, 3H), 3.25 (s, $6 \mathrm{H}), 3.41(\mathrm{~s}, 6 \mathrm{H}), 3.55(\mathrm{~s}, 2 \mathrm{H}), 3.58(\mathrm{~d}, \mathrm{~J}=5 \mathrm{~Hz}, 2 \mathrm{H}), 4.53(\mathrm{t}, \mathrm{J}=5 \mathrm{~Hz}, 1 \mathrm{H})$.

\section{H-3(6H)-Pyranon-4-nitrophenylhydrazon (20a)}

$0.77 \mathrm{~g}$ (5 mmol) 19a wurden unter Erwärmen in Ethanol gelöst und mit $0.5 \mathrm{~g}$ (5 mmol) 16 versetzt. Nach dem Abkühlen schieden sich gelbe und gelbrote Kristalle ab ( $E, Z$-Isomerenmischung), Schmp. 197-200 , Ausb. $0.66 \mathrm{~g}(57 \%) . \mathrm{C}_{11} \mathrm{H}_{11} \mathrm{~N}_{3} \mathrm{O}_{3}$ (233.2) Ber. C 56.6 H 4.75 N 18.0; Gef. C 56.7 H 4.76 N 17.9 Mol.-Masse 233 (ms). - IR: 1590, 1305, $126 \mathrm{~cm}^{-1}$. - ${ }^{1} \mathrm{H}-\mathrm{NMR}$ (d6-DMSO): $4.1-4.46$ (m, 2H), $4.28(\mathrm{~s})$ und $4.67(\mathrm{~s})$ (zusammen $2 \mathrm{H}$ ), $6.43(\mathrm{~s})$ und $6.75(\mathrm{t})$ und $7.03(\mathrm{t})$ (zusammen $2 \mathrm{H}$ ), 7.30 (dd, $\mathrm{J}=$ $9 / 1.5 \mathrm{~Hz}, 2 \mathrm{H}$ ), 8.24 (d, J $=9 \mathrm{~Hz}, 2 \mathrm{H}$ ), 10.27 (s) und 10.65 (s) (zusammen $1 \mathrm{H}$ ).

\section{H-3(6H)-Pyranon-semicarbazon (20b)}

Eine Mischung aus $0.5 \mathrm{~g}$ (5 mmol) 16, $0.56 \mathrm{~g}$ (5 mmol) 19b, $0.8 \mathrm{~g}$ Natriumacetat und $10 \mathrm{ml} 50$ proz. Ethanol wurde 10 min rückfließend erhitzt. Nach Zugabe von $10 \mathrm{ml}$ Wasser wurde das abgeschiedene, kristalline Produkt isoliert und aus Ethanol umkristallisiert. Farblose Kristalle (E,Z-Isomerenmischung), Schmp. 197-200 ${ }^{\circ}$, Ausb. 0.38 g (49\%). $\mathrm{C}_{6} \mathrm{H}_{9} \mathrm{~N}_{3} \mathrm{O}_{2}$ (155.2) Ber. C46.4 H 5.85 N 27.1; Gef. C 46.2 H5.96 27.3 Mol.-Masse 155 (ms). - IR: 1675, 1560, $1090 \mathrm{~cm}^{-1}$. - ${ }^{1} \mathrm{H}-\mathrm{NMR}$ (d6-DMSO): $4.0-4.3$ $(\mathrm{m}, 2 \mathrm{H}), 4.13$ (s) und 4.38 (s) (zusammen $2 \mathrm{H}$ ), 6.08-7.0 (m, 4H), $9.45(\mathrm{~s})$ und 9.8 (s) (zusammen 1H).

4a,10a-Dihydro-5,10-diphenyl-5,10-epoxy-1H-naphtho[2,3-b]pyran-4-(3H)-one (endolexo 22a222b)

a) Eine Mischung aus $0.98 \mathrm{~g}$ (10 mmol) 16, $1.35 \mathrm{~g}(5 \mathrm{mmol}) 21$ und $2 \mathrm{ml}$ Toluol wurde $3 \mathrm{~h}$ rückfließend erhitzt. Nach dem Abkühlen wurde mit Diethylether verdünnt und mit Wasser geschüttelt. Nach Trocknen über Natriumsulfat wurde die org. Phase i. Vak. eingeengt und der Rückstand fraktioniert aus Methanol kristallisiert. Die 1. Kristallfraktion wurde aus Methanol umkristallisiert. Farblose Kristalle (1. Diastereomer), Schmp. 131-132 , Ausb. 0.33 g (18 \%). $\mathrm{C}_{25} \mathrm{H}_{20} \mathrm{O}_{3}$ (368.4) Ber. C 81.5 H 5.47; Gef. C 81.6 H 5.46 Mol.-Masse 368 (ms). - IR: 1715, $1445 \mathrm{~cm}^{-1}$. - ${ }^{1} \mathrm{H}-\mathrm{NNMR}\left(\mathrm{CDCl}_{3}\right)$ : $2.82-4.04(\mathrm{~m}, \mathrm{SH}), 4.30(\mathrm{dd}, \mathrm{J}=10.5 / 5 \mathrm{~Hz}, 1 \mathrm{H}), 6.88-7.93(\mathrm{~m}, 14 \mathrm{H}) .-{ }^{13} \mathrm{C}-\mathrm{NMR}\left(\mathrm{CDCl}_{3}\right): 45.7$ (d), 53.9 (d), 67.6 (t), 73.6 (t), 89.4 (s), 90.5 (s), 121.1 (d), 121.9 (d), 126.7, 127.0, 127.3, 128.0, 128.4, $128.6,136.8$ (s), 136.9 (s), 145.3 (s), 146.5 (s), 208.3 (s). 
Die 2. Kristallfraktion uurde ebenfalls aus Methanol umkristallisiert. Farblose Kristalle (2. Diastereomer), Schmp. 135-137\%, Ausb. 0.21 g (11\%). $\mathrm{C}_{25} \mathrm{H}_{20} \mathrm{O}_{3}(368.4)$ Ber. C 81.5 H 5.47; Gef. C 81.2 H 5.59 Mol.-Masse 368 (ms). - IR: $1710,1455 \mathrm{~cm}^{-1} \cdot{ }^{-1} \mathrm{H}-\mathrm{NMR}\left(\mathrm{CDCl}_{3}\right): 2.63-4.03$ (m, $\left.3 \mathrm{H}\right), 3.70$ $(\mathrm{t}, \mathrm{J}=4 \mathrm{~Hz}, 1 \mathrm{H}), 3.80(\mathrm{~s}, 2 \mathrm{H}), 6.93-7.87(\mathrm{~m}, 14 \mathrm{H})$. - ${ }^{13} \mathrm{C}-\mathrm{NMR}\left(\mathrm{CDCl}_{3}\right): 47.0(\mathrm{~d}), 57.7(\mathrm{~d}), 66.4(\mathrm{t})$, $74.6(t), 91.0$ (s), 91.4 (s), 118.7 (d), 119.0 (d), 125.8, 126.1, 127.1, 128.2, 128.5, 128.8, 135.0(s), 135.9 (s), 147.1 (s), 147.7 (s), 208.1 (s).

b) $3.51 \mathrm{~g}$ (13 mmol) 21 und $1.91 \mathrm{~g}$ (11.5 mmol) 16 wurden unter Erwărmen in $3 \mathrm{ml}$ Toluol gelöst. Es wurde 36 h bei Raumtemp. gerührt, i. Vak. eingedampft und der Rückstand aus Methanol umkristallisiert. Farblose Kristalle (Diastereomerenmischung), Ausb. 4.39 (92\%).

\section{5,10-Diphenyl-2H-naphtho[2,3-c]pyran-4-(3H)-on (23)}

$2.76 \mathrm{~g}$ (7.5 mmol) 22a/226 wurden in $75 \mathrm{ml} 85$ proz. Phosphorsăure $15 \mathrm{~h}$ bei $50-60^{\circ}$ Badtemp. erwărmt. Dann wurde mit Wasser verdünnt und mehrfach mit Methylenchlorid extrahiert. Die vereinigten Extrakte wurden gewaschen, getrocknet (Natriumsulfat) und i. Vak. eingeengt. Der Rückstand wurde aus Methanol umkristallisiert. Blaßgelbe Kristalle, Schmp. 203-205\%, Ausb. 2.18 g (83 \%). $\mathrm{C}_{25} \mathrm{H}_{18} \mathrm{O}_{2}$ (350.4) Ber. C85.7 H 5.18; Gef. C 85.4 H5.10 Mol.-Masse 350 (ms). - IR: $1705,1560 \mathrm{~cm}^{-1}$.

- ${ }^{1} \mathrm{H}-\mathrm{NMR}\left(\mathrm{CDCl}_{3}\right): 4.27(\mathrm{~s}, 2 \mathrm{H}), 4.72(\mathrm{~s}, 2 \mathrm{H}), 7.08-7.75(\mathrm{~m}, 14 \mathrm{H})$.

\section{6,13-Dihydro-7,12-diphenyl-benz [6,7]isochromeno[4,3-b]indol (24)}

$0.7 \mathrm{~g}(2 \mathrm{mmol}) 23$ und $0.24 \mathrm{~g}$ (2.2 mmol) 6a wurden in $5 \mathrm{ml}$ Eisessig $20 \mathrm{~h}$ bei $60-70^{\circ}$ Badtemp. erwärmt. Nach Verdünnen mit Methanol wurde die Reaktionsmischung abgekühlt. Gelbe Kristalle, Schmp. 215-217 (Zers.), Ausb. 0.72 g (85\%, nicht umkristallisiert). $\mathrm{C}_{31} \mathrm{H}_{21} \mathrm{NO}$ (423.5) Ber. C87.9 H 5.00 N 3.3; Gef. C 87.9 H 5.06 N 3.3 Mol.-Masse 423 (ms). IR: $3450,1590 \mathrm{~cm}^{-1}$. ${ }^{1} \mathrm{H}-\mathrm{NMR}\left(\mathrm{CDCl}_{3}\right): 5.12$ (s, 2H), 6.33 (breit, $1 \mathrm{H}), 6.68-7.72(\mathrm{~m}, 18 \mathrm{H})$.

\section{Literatur}

1 108. Mitt. über Untersuchungen an Pyran-Derivaten; 107. Mitt.: F. Eiden und G. Patzelt, Arch. Pharm. (Weinheim) 318, 328 (1985).

2 C. A. Demerson, L. G. Humber, T. A. Dobson und R. R. Martel, J. Med. Chem. 18, 189 (1975); A. A. Asselin, L. G. Humber, Th. A. Dobson und J. Komlossy, J. Med. Chem. 19, 787 (1976).

3 A. A. Asselin, L. G. Humber und J. Komlossy, J. Med. Chem. 19, 792 (1976); L. G. Humber, C. A. Demerson, A. A. Asselin, M.-P. Charest und K. Pelz, Eur. J. Med. Chem. Chim. Ther. 10, 215 (1975).

4 Warner-Lambert Co (Erf. R. E. Brown und P. C. Unangst), U.S.P. 4028383 (7.6.77); C. A. 87, 10230111 (1977).

5 F. Eiden und K. Th. Wanner, Arch. Pharm. (Weinheim) 317, 958 (1984); F. Eiden und K. Th. Wanner, Liebigs Ann. Chem. 1984, 1759.

6 A. Croisy, A. Ricci, M. Janeevska, P. Jacquinon und D. Balucand, Chem. Lett. 1976, 5.

7 N. M. Sharkova, N. F. Kucherova und V. A. Zagorevskii, Khim. Geterotsikl. Soedin 1972, 81; C. A. $77,48303 t$ (1972).

8 J.E. Saxton in Indoles, Ed. A. Weissberger und E.C. Taylor, Vol. 4, J. Wiley, New York 1983.

9 K. Skinnemoen und K. Undheim, Acta. Chem. Scand. B 34, 295 (1980).

10 J. Gore und F. Guiques, Bull. Soc. Chim. Fr. 1970, 3521. 